\title{
Caracterização da Produção Brasileira em Artigos Científicos sobre Arte e Psicologia (2004-2014)
}

\author{
André Luiz Picolli da Silva - Universidade Federal do Sul e Sudeste do Pará, Marabá, Brasil \\ Terezinha de Camargo Viana - Universidade de Brasilia, Brasilia, Brasil
}

\begin{abstract}
Resumo
A Arte sempre fui objeto de estudo e instrumento de atuação para os profissionais de Psicologia no Brasil. Para ampliar o conhecimento sobre essa relação, o presente estudo tem por objetivo caracterizar como a Arte vem sendo utilizada por profissionais de Psicologia no país. O método utilizado foi o da investigação bibliográfica e exploratória em bases de dados sobre produção de artigos científicos referentes à utilização da Arte pela Psicologia no Brasil no período de 2004 a 2014. Selecionou-se 318 resumos de artigos que foram analisados a partir de seis categorias de análise: ano de publicação, tipo de estudo realizado, referencial teórico, tipo de manifestação artística, campo da Psicologia e objetivo do trabalho. Após a análise, conclui-se que no Brasil existe o predomínio de trabalhos teóricos e epistemológicos sobre artes Literárias e Visuais, com base na teoria psicanalítica, visando à ampliação teórico/conceitual.

Palavras-chave: arte, psicologia, Brasil, estado da arte, psicologia da arte
\end{abstract}

Characterization of the Brazilian production in scientific papers on Art and Psychology (2004-2014)

\begin{abstract}
Art has always been an object of study and performance tool for Psychology professionals in Brazil. In order to increase the knowledge on this relationship, the present study aims to characterize as Art has been used by psychology professionals in the country. The method used was the bibliographical and exploratory research in databases on production of scientific papers on the use Art by Psychology in Brazil from 2004 to 2014. 318 abstracts from six categories of analysis of articles that had been analyzed were selected: year of publication, type of study, theoretical references, artistic expression type, field in Psychology and objective of the work. After the analysis it was concluded that, in Brazil, there is a predominance of theoretical and epistemological work in literary and visual arts, based on psychoanalytic theory, aimed at theoretical / conceptual expansion. Keywords: art, psychology, Brazil, state of art, art psychology
\end{abstract}

Caracterización de la Producción Brasileña en Artículos Científicos sobre Arte y Psicología (2004-2014)

\begin{abstract}
Resumen
$\mathrm{El}$ arte ha sido siempre objeto de estudio e instrumento de práctica para los profesionales de Psicología en Brasil. Para ampliar el conocimiento sobre esta relación, el presente estudio tuvo por objetivo destacar como el Arte está siendo utilizado por los Psicólogos en Brasil. El método utilizado fue el de la investigación bibliográfica y exploratoria en bases de datos sobre la producción de artículos científicos referentes a la utilización del Arte por la Psicología en Brasil entre 2004 y 2014. Se seleccionaron 318 resúmenes de artículos que fueron analizados a partir de seis categorías: año de publicación, tipo de estudio realizado, referencia teórica, tipo de expresión artística, campo de la Psicología y objetivo del trabajo. Después del análisis se llegó a la conclusión de que en Brasil predominan los trabajos teóricos y epistemológicos sobre artes Literarias y Visuales, con base en la teoría psicoanalítica, destinado a la expansión teórico / conceptual.

Palabras clave: arte, psicología, Brasil, estado del arte, psicología del arte
\end{abstract}

A Arte é considerada por muitos autores, como Andriolo (2003), Paiva et al. (2004), Silva (2004) e Dionísio (2012), como uma das principais formas de expressão da humanidade. Outros estudiosos do tema, como Paglia (1992) e Paiva et al. (2004) sugerem que a Arte surgiu na aurora da humanidade como um desdobramento das primeiras manifestações religiosas, estando na base da cultura e confundindo-se com a própria noção de humanidade. Dada essa condição, não é de estranhar que a Arte tenha se convertido também em objeto de investigação para estudiosos clássicos do psiquismo humano, como, por exemplo: Freud (1907/1996), (1908/1996), (1910/1996) e (1914/1996), Jung (2001), Perls (1998) e Vygotski (1993) e (1998).
No Brasil, o pioneirismo de estudos relacionando Arte e fenômenos psicológicos, ocorreu com os trabalhos de Osório Cesar e Nise da Silveira que estudaram a relação entre Arte e Loucura, como afirmam Figueira, Amarante e Belancieire (2007) e Vasconcellos e Giglio (2007). Entretanto, para além dos estudos específicos entre Arte e loucura, é possível encontrar no país diversos trabalhos, como Moehlecke (2002), Silva (2004), Andriolo (2006a), Rivera (2006), Frayze-Pereira (2007) e (2011), Birman (2008) e Pereira (2012), que buscam discutir de modo mais amplo a relação entre manifestações artísticas e processos de saúde, de desenvolvimento social, desenvolvimento humano, da personalidade, de educação, etc. 
Indo mais além, existem trabalhos com objetivo de ampliar a compreensão da relação entre Arte e a Ciência Psicológica contribuindo assim para a estruturação do campo da Psicologia da Arte no país, como, por exemplo, Frayze-Pereira (1994), Vivian e Trindade (2003), Andriolo (2003) e (2006a), Moutinho e Conti (2010) e Tardivo (2012). Entretanto, apesar de existir muitos trabalhos nessa área, não se sabe ao certo quais as características do campo da Psicologia da Arte no país.

Durante a realização do trabalho de doutorado de um dos autores, evidenciou-se que não existem pesquisas no Brasil que identifiquem quais as principais teorias, campos de atuação ou tipos de trabalho que são realizados pelos psicólogos brasileiros utilizando a arte. Constando-se essa carência, decidiu-se realizar uma pesquisa que pudesse suprir essa lacuna encontrada sobre as práticas profissionais dos psicólogos brasileiros, sendo esta a principal justificativa para a realização deste trabalho. Nesta perspectiva, a presente pesquisa tem por objetivo principal caracterizar como ocorreu a relação entre a Arte e a Psicologia no Brasil, entre os anos de 2004 a 2014.

A relevância deste trabalho consiste no fato de que conhecer como os psicólogos do país tem se apropriado da Arte, tanto para estudos, quanto para suas práticas profissionais contribui para que, como afirmam Silva (2004) e Ferreira (2002), seja possível identificar as lacunas na construção da ciência e auxiliar seu desenvolvimento em prol de uma melhor qualidade de vida para todos. Nessa perspectiva, buscou-se nesse estudo obter uma visão ampliada da relação entre Arte e Psicologia, não sendo feita distinção, durante a coleta de dados, entre trabalhos que tenham utilizado a Arte como instrumento de prática profissional do psicólogo ou trabalhos que a tenham utilizado como objeto de estudo.

Com base nisso, a escolha metodológica para a concretização deste estudo, foi a realização de uma de pesquisa caracterizada como "estado da Arte", pois de acordo com Laville e Dionne (1999) e Luna (2002), tais pesquisas são as mais indicadas para caracterizar a situação atual do entendimento da ciência sobre um determinado fenômeno. Pesquisas bibliográficas tradicionalmente conhecidas como pesquisas sobre o estado da Arte têm por principal objetivo fazer o "mapeamento" da produção científica sobre determinado tema em um lugar e período de tempo específico.

A importância da realização de trabalhos bibliográficos desse tipo, como salienta Ferreira (2002), reside no fato de que, para avançar de modo seguro, a Ciência precisa ter conhecimento das características das produções que estão sendo realizadas nas mais diversas áreas. Para não correr o risco de repetir o que já foi descoberto anteriormente, é necessário ter uma boa noção das perspectivas sob as quais os fenômenos estão sendo estudados, de modo que seja possível identificar com clareza as tendências, lacunas, incoerências ou contradições do conhecimento que está sendo produzido. Assim sendo, uma pesquisa sobre o estado da Arte, como a que foi realizada, também demonstra sua relevância por auxiliar a ciência a avançar com maior segurança no conhecimento sobre os aspectos da relação entre Psicologia e Arte no Brasil, auxiliando futuros pesquisadores e profissionais da área a realizarem seus trabalhos.

\section{Método}

O presente estudo se caracteriza como uma pesquisa de base, conforme Marconi e Lakatos (1986); bibliográfica, conforme Laville e Dionne (1999) e Carvalho (1989), e de caráter descritivo e exploratório, conforme Gil (1991), sobre o estado da Arte da produção científica relacionada à utilização da Arte pela Psicologia no Brasil no período correspondente entre os anos de 2004 a 2014. Nesse sentido, este estudo prima por aumentar a familiaridade com o fenômeno estudado, tornando-o mais explícito a fim de facilitar a formulação de futuras hipóteses ele, além de determinar suas características descrevendo as diversas variáveis que o compõem, como argumentam Nunes e Huntz (2014).

Nessa mesma perspectiva, Luna (2002) enfatiza que as pesquisas sobre o estado da Arte têm por objetivo descrever a situação atual que se encontra uma determinada área de pesquisa, ou mesmo os estudos realizados sobre algum fenômeno específico e enfatiza que tais pesquisas são excelentes fontes de atualização para pesquisadores que pertencem a outras áreas do conhecimento. Tais características demonstram que essas pesquisas são, sobretudo, fundamentais para auxiliar no avanço do conhecimento científico sobre determinado tema ou fenômeno, como apregoa Ferreira (2002).

Assim sendo, tendo tais concepções como princípios norteadores para a concretização deste estudo, além do exposto, também foram considerados como fontes para embasamento metodológico, os trabalhos realizados por Zanella e Titon (2005), Mazer e 
Melo-Silva (2010) e Silva e Nakano (2012), devido à proximidade dos estudos realizados por essas autoras com o objeto e o tipo de pesquisa ora proposta.

\section{Fontes de Informação}

Tendo por base os trabalhos supracitados, foram utilizadas como fontes de informação, bases de dados eletrônicas consideradas como referência dentro da área da Psicologia, a saber: PePSIC, SciELO, LILACS e IndexPSI periódicos técnico-ciêntíficos.

\section{Seleção das Fontes de Informação}

As fontes de informação já citadas foram selecionadas devido a sua grande reputação dentro da área das Ciências Humanas em geral, e da Psicologia em especial, sendo reconhecidas como fontes confiáveis e de grande relevância, como atestam os trabalhos de Zanella e Titon (2005), Mazer e Melo-Silva (2010), Koerich e Erdmann (2011) e Silva e Nakano (2012).

\section{Instrumentos}

Para realizar o procedimento de coleta de informações foram utilizados como instrumentos, computador com acesso a internet e folhas de registro contendo um quadro geral criado especificamente para sistematizar e organizar as informações coletadas. O quadro geral consistia em um quadro contendo seis categorias de análise previamente estabelecidas antes do início da coleta de dados, a saber: ano de publicação, tipo de estudo realizado, referencial teórico utilizado, tipo de manifestação artística estudada/utilizada, campo da Psicologia em que a Arte foi utilizada e objetivo do trabalho realizado. Essas categorias de análise foram estabelecidas, tomando-se como base as categorias utilizadas nos trabalhos de Zanella e Titon (2005) e Silva e Nakano (2012), sendo semelhantes às categorias utilizadas por esses autores para caracterizar o estado da Arte da produção científica brasileira sobre o fenômeno da criatividade.

\section{Procedimentos}

Para identificação dos trabalhos referentes à relação entre Arte e Psicologia no Brasil no período entre 2004 e 2014 foram acessadas as bases de dados apresentadas no item Fontes de Informação e, em seguida, realizada uma busca utilizando-se como único descritor a palavra-chave "Arte". Optou-se pela utilização de uma única palavra-chave, pois, de acordo com a literatura utilizada para embasar metodologicamente essa pesquisa, um dos elementos que dificultam a localização de trabalhos em bases de dados é a utilização de descritores que podem eliminar trabalhos que, apesar de apresentarem palavras-chaves diferentes, tratam das mesmas temáticas. Assim, por exemplo, se fosse utilizada a combinação das palavras-chaves "Arte" + "Psicologia" correr-se-ia o risco de não localizar trabalhos que podem ser da área de Psicologia, mas que utilizaram outros descritores como "Arte" + "Psicanálise", "Arte" + "Construtivismo" ou "Arte" + "Gestalt".

Com base nisso, foi utilizada a palavra-chave considerada central para a pesquisa, de modo a que se obtivesse uma maior amplitude de artigos passíveis de análise. Assim sendo, foram identificadas um total de 5.203 (cinco mil duzentos e três) artigos publicados no Brasil entre 2004 e 2014 que estavam relacionados com a temática da Arte.

A partir da quantidade de artigos identificados, foi realizado um refinamento das informações coletadas, por meio da leitura de todos os resumos dos artigos encontrados a fim de identificar e selecionar somente os resumos que fizessem referencia a trabalhos que relacionassem a Arte e a Psicologia, sendo que os demais resumos que não apresentaram essa característica foram descartados.

Após a realização desse procedimento, foram identificados em um total de 318 (trezentos e dezoito) artigos publicados entre 2004 e 2014 relacionando a Arte e a Psicologia. Em seguida, foi realizada uma nova leitura dos 318 (trezentos e dezoito) resumos selecionados de modo que eles pudessem ser organizados em um quadro geral com as seis categorias de análise já informadas. As informações contidas em cada uma dessas categorias foram posteriormente transferidas para gráficos e quadros específicos, para que pudessem ser analisadas a partir da confrontação dos dados ali existentes com dados encontrados em artigos relacionados a temática estudada.

\section{Resultados e Discussão}

A apresentação e discussão dos resultados fazem referência aos 318 (trezentos e dezoito) resumos de artigos analisados, que foram selecionados por serem trabalhos referentes a estudos, relatos de experiência, experimentos, reflexões, etc., relacionando a Arte e a Psicologia. Será apresentado a seguir o número total de trabalhos, bem como suas porcentagens dentro de cada categoria de análise, de modo a auxiliar a visualização dos dados e o entendimento dos aspectos que mais se destacaram em cada categoria. 
Na Figura 1, é apresentada a quantidade de ocorrência entre os anos de 2004 e 2014 de artigos publicados envolvendo Arte e Psicologia. É possível evidenciar um maior número de publicações no ano de 2008, quando 47 artigos (14,78\%) foram publicados. Também se destacam os anos de 2006 e 2009, ambos com 39 artigos (12,26\%), o ano de 2010 com 37 artigos (11,64\%) e os anos de 2007 e 2011, ambos com 28 artigos (8,81\%).

Analisando a Figura 1, é interessante perceber que entre os anos 2004 a 2008 foi ocorrendo um aumento nas publicações de artigos na área de Psicologia de trabalhos relacionados à Arte, com exceção do ano de 2007. A partir do ano de 2008, no qual ocorreu o ápice, o número de publicações foi gradualmente decaindo até o ano de 2013, chegando a menor quantidade de ocorrência por ano com um total de 13 artigos, o que corresponde a 4,09\% da amostra estudada. Entretanto, no ano de 2014 houve uma retomada de interesse na publicação de trabalhos nessa temática, visto ter ocorrido um aumento para 21 artigos publicados o que corresponde a $6,60 \%$ da amostra estudada.

Com base apenas na leitura dos resumos, não foi possível identificar o que pode ter causado essa oscilação na quantidade de publicações, sendo que o máximo que se poderia fazer para tentar explicar esse fenômeno seriam especulações. $\mathrm{O}$ que se pode constatar ao observar a Figura 1, é que desde 2008 houve uma tendência de diminuição das publicações nessa temática. Entretanto, apenas com base nessa observação, não se pode afirmar que houve um desinteresse por parte dos psicólogos em utilizar a Arte em suas práticas profissionais. Uma possível explicação para esse fenômeno, é que o aumento do rigor para a publicação de trabalhos em revistas científicas no país, que vem ocorrendo nos últimos anos, pode ter dificultado aos profissionais que possuem pouca habilidade na escrita científica em publicar o relato de seus trabalhos nesses periódicos. Considera-se isso, pois na maioria dos resumos analisados identificou-se que a escrita deles tende mais à escrita poética do que técnica o que, por vezes, pode dificultar a publicação em periódicos que enfatizam a escrita científica.

Na Figura 2, é apresentada a quantidade de ocorrência dos tipos de trabalhos publicados envolvendo Arte e Psicologia entre os anos de 2004 e 2014. É possível observar que a maioria dos trabalhos publicados nesse período, ou seja, 92 artigos (29\%) tratam de estudos reflexivos sobre a Arte, realizados na área da Psicologia.

Além de estudos reflexivos, também foi possível encontrar nesse período de 10 anos, 67 artigos (21\%) de estudos sobre teorias ou aspectos teóricos que envolvem a Arte e a Psicologia, 41 artigos (13\%) sobre relatos de experiência profissional da utilização da Arte na prática profissional do psicólogo, 20 artigos $(6 \%)$ sobre estudos de caso envolvendo a Arte, 17 artigos (5\%) sobre pesquisas exploratórias e 03 artigos (1\%) sobre pesquisas experimentais envolvendo a Arte. Outro ponto interessante a ser destacado na Figura 2 é que em 78 artigos (25\%) os resumos não apresentavam indicação sobre o tipo de trabalho que foi realizado, não sendo possível identificar assim a que tipo de trabalhos esses artigos faziam referência.

Na Figura 2, fica evidenciado que a maioria dos trabalhos realizados no país entre os anos de 2004 e 2014 sobre Arte e Psicologia encontra-se na categoria de trabalhos reflexivos e teóricos. É interessante destacar que esse é um resultado muito semelhante ao encontrado por Nakano e Wechsler (2007) e Silva e Nakano (2012)

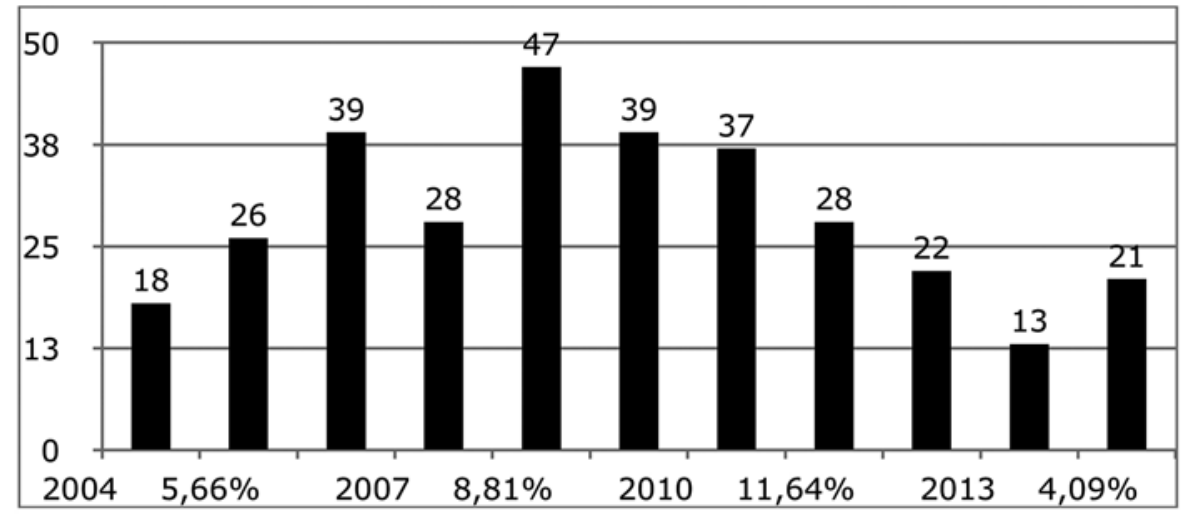

Figura 1. Quantidade de publicações por ano. 
em estudos realizados sobre o fenômeno da criatividade, que é uma temática próxima à temática da Arte, o que corrobora que essa é uma tendência no país para os estudos dessa área.

De acordo com Laville e Dionne (1999), o predomínio de trabalhos de cunho teóricos sobre trabalhos de cunho prático em uma determinada área são um indicativo de que essa área encontra-se mais em fase de estruturação e de definição conceitual do que de aplicação do conhecimento. É claro que a quantidade de trabalhos teóricos ou práticos dependerá, sobretudo, da natureza do fenômeno que está sendo estudado, mas, em se tratando da Arte e da sua relação com a Psicologia no Brasil, a constatação de Laville e Dionne (1999) parece fazer sentido. $\mathrm{Na}$ leitura realizada dos resumos para se obter os dados apresentados na Figura 2, evidenciou-se que a maioria dos trabalhos buscava ampliar o entendimento de conceitos ou trabalhar teoricamente aspectos relacionados a Arte. Nesse sentido, constata-se que a grande variabilidade de tipos de Arte existentes, associada à diversidade de referenciais epistemológicos utilizados pela Psicologia, faz com que a relação entre esses dois fenômenos ainda seja predominantemente no âmbito teórico, demonstrando assim, como indicam Laville e Dionne (1999), que o campo da Psicologia da Arte ainda se configura como um campo novo dentro da Psicologia brasileira.

Na Figura 3, é apresentada a quantidade de ocorrência dos referenciais teóricos utilizados nos artigos publicados envolvendo a relação entre Arte e Psicologia entre os anos de 2004 e 2014. De acordo com a Figura 3, a maioria dos trabalhos que foram publicados sobre Psicologia e Arte nesse período no Brasil utilizou a Psicanálise como referência teórica, perfazendo um total de 188 artigos publicados, o que corresponde a 56, 96\% do total de trabalhos analisados.

Além do referencial psicanalítico, também houve ocorrência de trabalhos realizados com os seguintes referenciais teóricos: Psicologia Histórico-Cultural, com ocorrência de 16 artigos (4,85\%); Psicologia

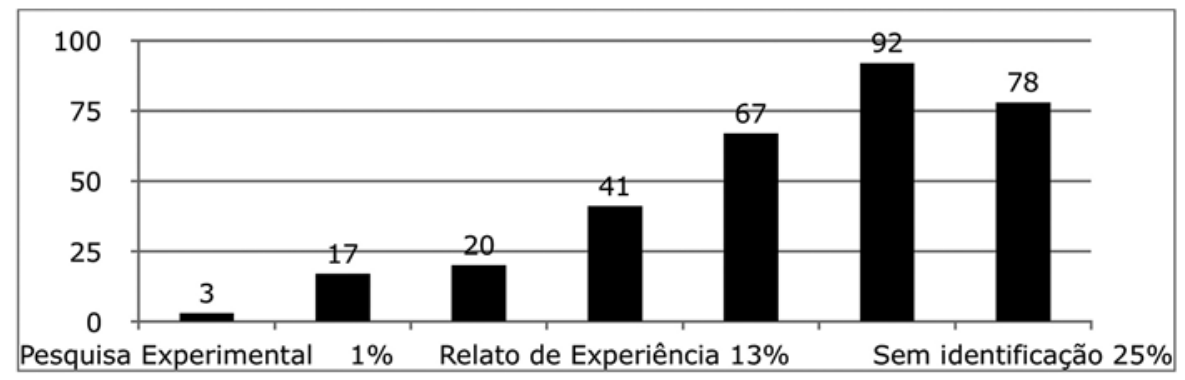

Figura 2. Tipo de trabalho relatado nos artigos.

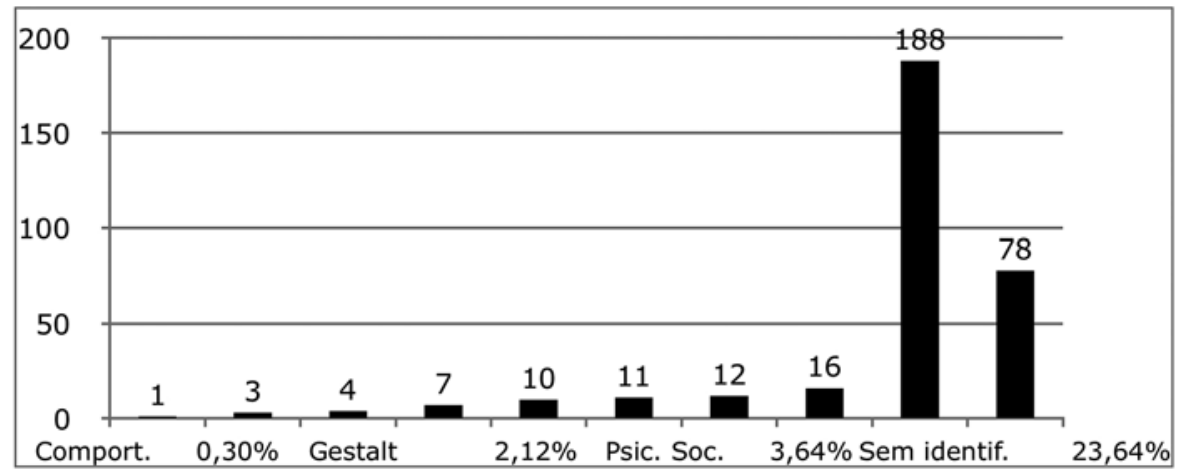

Figura 3. Referenciais teóricos utilizados.

Obs.: O número total dos tipos de referencias teóricos encontrados não corresponde ao número total de artigos, pois em muitos trabalhos foram utilizados mais de um referencial teórico. 
Social, com ocorrência de 12 artigos (3,64\%); Fenomenologia, com ocorrência de 11 artigos (3,34\%); Psicologia Analítica, com ocorrência de 10 artigos (3,04\%); Psicologia da Gestalt, com ocorrência de 07 artigos (2,12\%); Teoria Crítica da Sociedade, com ocorrência de 04 artigos (1,21\%); Psicodrama, com ocorrência de 03 artigos $(0,90 \%)$ e Comportamentalismo, com ocorrência de 01 artigo $(0,30 \%)$. Ainda é interessante destacar na Figura 3 que, em 78 artigos $(23,64 \%)$ da amostra observada, os resumos não apresentavam indicação alguma sobre qual referencial teórico foi utilizado para a realização dos trabalhos ou investigações realizadas.

$\mathrm{Na}$ Figura 3, fica clara a forte utilização do referencial psicanalítico na realização de trabalhos envolvendo a Arte e a Psicologia. Apesar de em um primeiro momento parecer surpreendente o grande número de trabalhos utilizando esse único referencial teórico, tal resultado vai de encontro ao que foi encontrado em trabalhos como os de Alencar e Fleith (2003), e Nakano e Wechsler (2007) que encontraram uma predominância do referencial teórico psicanalítico nos trabalhos realizados sobre criatividade.

Uma explicação para a ocorrência desse predomínio vem do fato de que a maioria dos artigos referentes a estudos teóricos e trabalhos reflexivos, apresentados na Figura 2, utilizava a Literatura como objeto de estudo ou intervenção. Tal configuração explica a marcante presença da Psicanálise como principal referencial teórico, pois ela apresenta forte ligação com a Literatura como enfatizam Raffaelli (1996) e Teixeira (2005). De qualquer modo, o que ficou evidenciado pela Figura 3 é que os referenciais teóricos que mais se destacaram foram a Psicanálise, a Psicologia Histórico-Cultural e a Psicologia Social, o que também vai de encontro aos resultados obtidos em um estudo sobre a criatividade realizado por Zanella \& Titon (2005).

$\mathrm{Na}$ Tabela 1, são apresentados os tipos e a quantidade de ocorrência das manifestações artísticas encontradas nos resumos dos artigos publicados envolvendo a relação entre Arte e Psicologia entre os anos de 2004 e 2014. Nesta figura destacam-se a ocorrência da Literatura em 54 artigos (15,74\%); das Artes Plásticas em 42 artigos (12,24\%); do cinema/ vídeo em 28 artigos (8,16\%); e do estudo sobre artistas em 22 artigos (6,42\%). Também se destaca na Tabela 1, o fato de que $113(32,94 \%)$ dos resumos observados na amostra coletada não faziam referência alguma ao tipo de manifestação artística que foi estudada/utilizada nos trabalhos publicados.
Na Tabela 1, constata-se que existe uma grande gama de manifestações artísticas que são utilizadas por psicólogos em suas práticas profissionais ou na realização de suas pesquisas. Tal configuração corrobora com o que se encontra na literatura, autores como Moehlecke (2002), Andriolo (2003) e Reis (2014), destacam em seus trabalhos que a Psicologia brasileira utiliza a Arte nos seus mais diversas variações, aspectos, modos e formas de manifestação, não se prendendo apenas aos modelos já consagrados de Arte, como a Pintura, a Literatura, a Escultura ou o Teatro.

Outro ponto interessante a ser destacado é que o alto índice de trabalhos sobre Literatura, Artes Plásticas, Cinema e estudos sobre artistas parece ter uma relação direta com o predomínio do referencial psicanalítico no campo da Psicologia da Arte, como visto na Figura 3. Tal teoria como demonstram os trabalhos de Raffaelli (1996), Teixeira (2005) e Dionísio (2012), privilegiam o estudo de elementos como a palavra, o olhar e a história de vida, o que estimularia a realização de trabalhos ou estudos sobre manifestações artísticas que destacam esses elementos.

$\mathrm{Na}$ Tabela 2, é apresentada a quantidade de trabalhos por campo de atuação dentro a área da Psicologia nos quais a Arte foi utilizada como principal instrumento de intervenção ou objeto de estudo entre os anos de 2004 e 2014. Nesta Tabela, destacam-se o campo da Epistemologia com 81 artigos publicados (22,38\%); o campo da Psicologia da Arte com 51 artigos publicados $(14,09 \%)$; o campo da clínica com 29 artigos (8,01\%); o campo da saúde metal com 22 artigos (6,08\%); e o campo social/comunitário com 21 artigos (5,80\%). Outra informação que também se destaca na Tabela 2 é que, em 74 dos resumos observados $(20,44 \%)$, não havia referência alguma sobre em qual campo a Arte foi utilizada, não sendo possível identificar sobre quais campos de atuação os artigos se referiam.

A Tabela 2 mostra que apesar de existir uma grande variedade nos campos de atuação que utilizam a Arte, a maioria dos trabalhos encontra-se no campo epistemológico (81 artigos), seguido pelo campo da Psicologia da Arte, no tocante especificamente a interpretação de obras de Arte (51 artigos). Isso mostra que a ênfase dada os trabalhos que relacionam Arte e Psicologia encontram-se realmente no âmbito dos trabalhos reflexivos e teóricos, fato esse que é corroborado pelos dados apresentados na Figura 2.

A grande quantidade de trabalhos epistemológicos e teóricos parece mostrar um padrão na produção 
Tabela 1.

Tipos de manifestações artísticas que foram objeto dos artigos.

\begin{tabular}{lcc}
\hline Tipos de Manifestação Artística & Quant. de Artigos & $\mathbf{\%}$ \\
\hline Literatura & 54 & $15,74 \%$ \\
Artes plásticas (pinturas e desenhos) & 42 & $12,24 \%$ \\
Cinema/vídeos & 28 & $8,16 \%$ \\
Estudos sobre artistas & 22 & $6,42 \%$ \\
Teatro & 19 & $5,54 \%$ \\
Música & 17 & $4,97 \%$ \\
Arte urbana & 10 & $2,92 \%$ \\
Poesia & 07 & $2,04 \%$ \\
Fotografia & 06 & $1,76 \%$ \\
Escultura & 06 & $1,76 \%$ \\
Dança & 03 & $0,87 \%$ \\
Instalações & 03 & $0,87 \%$ \\
Artesanato & 03 & $0,87 \%$ \\
Arte Digital & 02 & $0,58 \%$ \\
Circo & 02 & $0,58 \%$ \\
Gastronomia & 01 & $0,29 \%$ \\
Canto & 01 & $0,29 \%$ \\
Arte rupestre & 01 & $0,29 \%$ \\
Arte corporal & 01 & $0,29 \%$ \\
Histórias em quadrinho & 01 & $0,29 \%$ \\
Crítica de arte & 01 & $0,29 \%$ \\
Sem identificação & 113 & $32,94 \%$ \\
\hline Total & $\mathbf{3 4 3}$ & $\mathbf{1 0 0 \%}$ \\
\hline
\end{tabular}

Obs.: O número total de tipos de manifestações artísticas estudadas não corresponde ao número total de artigos, pois em muitos artigos faziam referência a mais de um tipo de manifestação.

dos trabalhos sobre Psicologia e Arte que é a busca por uma estruturação conceitual e metodológica. Essa busca por modos específicos de estudar a Arte está presente no campo da Psicologia da Arte no Brasil desde seu início, como atesta Andriolo (2006a) ao realizar um estudo histórico sobre a metodologia utilizada no campo da Psicologia da Arte no País. Nesse sentido, os dados apresentados na Tabela 2 vão de encontro ao que foi observado por Andriolo (2006a) e indicam que essa busca por definições epistemológicas parece ser uma tendência contínua nesse campo.

$\mathrm{Na}$ Tabela 3, são apresentados os objetivos encontrados nos resumos dos trabalhos publicados entre 2004 e 2014 dentro da área da Psicologia nos quais a Arte foi utilizada como principal instrumento de intervenção ou objeto de estudo. Nesta Tabela, destacam-se como principais objetivos encontrados a ampliação teórico/conceitual com 73 artigos publicados (19,90\%); a discussão de temas sobre Arte com 66 artigos publicados (17,98\%); a ampliação da prática profissional, com 42 artigos (11,45\%); o autoconhecimento, com 25 artigos (6,81\%); e o desenvolvimento social/comunitário com 20 artigos (5,45\%). Outra informação que também se destaca na Tabela 3 é que, em 109 dos resumos observados (29,70\%), não havia referência sobre quais eram os objetivos dos trabalhos, não sendo possível identificar com clareza quais eram as finalidades dos artigos publicados.

Como é possível observar na Tabela 3, a maioria dos objetivos dos trabalhos que foram encontrados 
Tabela 2.

Campo de atuação da psicologia em que a arte foi utilizada.

\begin{tabular}{|c|c|c|}
\hline Campo de Atuação & Quant. de Artigos & $\%$ \\
\hline Epistemologia/Estudos estéticos & 81 & $22,38 \%$ \\
\hline Psicologia da arte (interpretação de obras) & 51 & $14,09 \%$ \\
\hline Clínica & 29 & $8,01 \%$ \\
\hline Saúde mental & 22 & $6,08 \%$ \\
\hline Social/comunitário & 21 & $5,80 \%$ \\
\hline Desenvolvimento humano & 15 & $4,14 \%$ \\
\hline Educação & 12 & $3,31 \%$ \\
\hline Formação profissional & 09 & $2,49 \%$ \\
\hline Estudos históricos & 08 & $2,20 \%$ \\
\hline Organizacional/trabalho & 07 & $1,93 \%$ \\
\hline Hospitalar & 06 & $1,65 \%$ \\
\hline Terceira Idade & 05 & $1,39 \%$ \\
\hline Psicopatologia & 05 & $1,39 \%$ \\
\hline Saúde & 04 & $1,10 \%$ \\
\hline Estudos culturais & 03 & $0,83 \%$ \\
\hline Família & 03 & $0,83 \%$ \\
\hline Adolescência & 03 & $0,83 \%$ \\
\hline Avaliação Psicológica & 02 & $0,55 \%$ \\
\hline Jurídico & 01 & $0,28 \%$ \\
\hline Aprendizagem & 01 & $0,28 \%$ \\
\hline Sem identificação & 74 & $20,44 \%$ \\
\hline Total & 362 & $100 \%$ \\
\hline
\end{tabular}

Obs.: O número total de Campos de Atuação em que a Arte foi utilizada não corresponde ao número total de artigos, pois em alguns trabalhos a Arte fui utilizada em mais e um Campo.

Tabela 3 .

Objetivos dos trabalhos analisados.

\begin{tabular}{lcc}
\hline Objetivos & Quant. de Artigos & \% \\
\hline Ampliação teórico/conceitual & 73 & $19,90 \%$ \\
Discussão de temas sobre arte & 66 & $17,98 \%$ \\
Ampliação da prática profissional & 42 & $11,45 \%$ \\
Autoconhecimento & 25 & $6,81 \%$ \\
Desenvolvimento social/comunitário & 20 & $5,45 \%$ \\
Caracterização de técnicas & 12 & $3,27 \%$ \\
Tratamento psicoterápico & 10 & $2,72 \%$ \\
Ampliação da formação profissional & 10 & $2,72 \%$ \\
Sem identificação & 109 & $29,70 \%$ \\
\hline Total & $\mathbf{3 6 7}$ & $\mathbf{1 0 0} \%$ \\
\hline
\end{tabular}

Obs.: O número total de Objetivos encontrados nos trabalhos estudados não corresponde ao número total de artigos, pois muitos trabalhos apresentavam mais de um objetivo. 
dizem respeito à ampliação teórico/conceitual e discussão sobre temas relacionados a Arte, seguidos por uma busca da ampliação da prática profissional. Tal predomínio de objetivos demonstra uma preocupação por parte dos pesquisadores e profissionais em ampliar sua compreensão sobre o tema Arte e de como este pode ser relacionado com o saber científico e a prática profissional na Psicologia.

Essa configuração vai de encontro direto aos trabalhos de Andriolo (2006b) e Silva (2004) que argumentam sobre a existência de uma carência na formação dos psicólogos brasileiros que durante sua graduação tem pouco contato com a Arte. É pouco comum que estudantes de Psicologia nas universidades brasileiras tenham práticas de ensino voltadas à compreensão de fenômenos estéticos, sendo mais comum que os estudantes aprendam a utilizar manifestações artísticas como instrumentos indiretos para intervenções profissionais, como salienta Silva (2004). Nesse sentido, o alto grau de ocorrência de trabalhos visando à ampliação teórico/conceitual pode, além de indicar que esse campo da Psicologia ainda encontra-se em franca fase de estruturação, indicar uma carência na formação dos psicólogos no tocante a Psicologia da Arte, o que pode fazer com que estes, enquanto profissionais, busquem realizar pesquisas para suprir as lacunas existentes nessa formação, como alertaram Andriolo (2006b) e Silva (2004).

\section{Considerações Finais}

Com a realização deste trabalho, objetivou-se caracterizar como vem ocorrendo a relação entre Psicologia e Arte no país no período entre 2004 e 2014 com base em três grandes grupos de análise: a evolução temporal de ocorrência dessa relação (publicações por ano); os procedimentos metodológicos mais utilizados no Brasil (tipo de estudo realizado - referencial teórico utilizado - tipo de manifestação artística utilizada/ estudada); e o principal foco dos estudos/trabalhos feitos com/sobre Arte pela Psicologia no Brasil (campo da Psicologia no qual a Arte foi utilizada/estudada objetivos dos trabalhos). Apesar de essa caracterização ter sido realizada, é importante frisar que este estudo apresenta como uma limitação metodológica o fato de ter sido utilizada como fonte de informação apenas os resumos de artigos publicados em revistas científicas brasileiras, não sendo analisadas outras produções como dissertações, teses, livros ou comunicações em congressos científicos.
Nesse sentido, resta a pergunta: será que as características da produção brasileira envolvendo Arte e Psicologia encontradas em resumos de artigos científicos correspondem a características existentes em outros meios de divulgação científica? Com base nessa provocação, constata-se que existem desdobramentos necessários a esta pesquisa, ficando o convite aos pesquisadores da área para ampliarem o estudo aqui realizado. Outra problemática encontrada em relação à escolha metodológica deste trabalho, ou seja, a análise de resumos de artigos, foi a quantidade de resumos encontrados com uma escrita inadequada para trabalhos científicos. Muitos resumos não apresentavam de modo claro, elementos, como os objetivos, métodos, resultados ou conclusões, o que tornou a análise mais trabalhosa. Tal situação é preocupante não apenas por tornar difícil a leitura de textos científicos produzidos no país, mas também por demonstrar que muitos profissionais brasileiros têm dificuldade em realizar a escrita científica, o que pode indicar que esses profissionais também tenham dificuldade de aplicar o método científico em suas práticas profissionais.

Por fim, é importante frisar também que os resultados encontrados nesta pesquisa se constituem como um marco para a compreensão de como vem se estabelecendo a relação entre Arte e Psicologia no país. Enfatiza-se essa colocação, pois, durante a realização da pesquisa, não foram encontrados trabalhos que fornecessem um panorama geral sobre o "estado da Arte" das produções brasileiras envolvendo essas duas áreas. Tal ineditismo, de certo modo dificultou a análise dos dados coletados, visto não existir parâmetros para comparar as informações encontradas como outros trabalhos específicos sobre Psicologia e Arte, tendo as análises sido realizadas com fenômenos próximos ao objeto de estudo em questão, como, por exemplo, a criatividade. Entretanto, apesar dessas dificuldades e das limitações metodológicas mencionadas, conclui-se que a produção brasileira sobre Arte e Psicologia no período entre 2004 e 2014 apresenta a característica de ser uma produção voltada para trabalhos teóricos de cunho epistemológico, dentro de uma perspectiva teórica psicanalítica, visando à ampliação da compreensão de conceitos, com predomínio de trabalhos voltados às artes literárias e visuais.

\section{Referências}

Alencar, E. M. L. S., \& Fleith, D. S. (2003). Contribuições teóricas recentes ao estudo da criatividade. 
Psicologia: Teoria e Pesquisa, 19(1), 1-8. doi: 10.1590/ S0102-37722003000100002

Andriolo, A. (2003). A "Psicologia da Arte" no olhar de Osório Cesar: Leituras e escritos. Psicologia Ciência e Profissão, 23(4), 74-81. doi: 10.1590/ S1414-98932003000400011

Andriolo, A. (2006a). O método comparativo na origem da Psicologia da Arte. Psicolo-gia USP, 17(2), 43-57. doi: 10.1590/S0103-65642006000200003

Andriolo, A. (2006b). O silêncio da pintura ingênua nos ateliêspsiquiátricos. Psicolo-gia: Teoriae Pesquisa, 22(2), 227-232. doi: 10.1590/S0102-37722006000200012

Birman, J. (2008). Criatividade e sublimação em Psicanálise. Psicologia Clínica, 20(1), 11-26. doi: 10.1590/ S0103-56652008000100001

Carvalho, M. C. M. (Org.). (1989). Construindo o Saber - Metodologia Científica: fundamentos e técnicas. Campinas: Papirus.

Dionísio, G. H. (2012). A Psicologia do ego de Ernst Kris e seu legado para a "Psicaná-lise da Arte". Psicologia USP, 23(1), 191-209. doi: 10.1590/ S0103-65642012000100010

Ferreira, N. S. de A. (2002). As pesquisas denominadas "Estado da Arte". Educação e Sociedade, 23(79), 257272. doi: 10.1590/S0101-73302002000300013

Figueira, E., Amarante, M. C., \& Belancieiri, M. F. (2007). O pioneirismo como espe-lho: O uso da arte por psicólogos em ambientes hospitalares. Psicologia hospitalar, 5(1), 100-113. Recuperado de http://pepsic.bvsalud.org/scielo.php?script $=$ sci_ arttext\&pid $=$ S167774092007000100007\&lng $=$ pt $\& \operatorname{lng}=$ pt.

Frayze-Pereira, J. A. (1994). A alteridade da arte: Estética e psicologia. Psicologia USP, 5(1/2), 35-60. Recuperado de http://pepsic.bvsalud.org/pdf/ psicousp/v5n1-2/a04v5n12.pdf

Frayze-Pereira, J. A. (2007). Da arte de interpretar o paciente como obra de arte. Jornal de Psicanálise, 40(73), 133-144. Recuperado de http://pepsic. bvsalud.org/pdf/jp/v40n73/v40n73a12.pdf

Frayze-Pereira, J. A. (2011). Arte e inveja: Relações entre amor e ódio, clínica e política na era do vazio. Ide, 34(52), 157-171. Recuperado de http://pepsic.bvsalud.org/scielo.php?script=sci_arttext\&pid =S0101-31062011000100016
Freud, S. (1907/1996). Delírios e Sonhos na Gradiva de Jensen. Edição Standart Brasileira das Obras Completas de Sigmund Freud. Vol. IX. Rio de Janeiro: Imago.

(1908/1996). Escritores Criativos e Devaneio. Edição Standart Brasileira das Obras Completas de Sigmund Freud. Vol. IX. Rio de Janeiro: Imago.

(1910/1996). Leonardo da Vinci e uma lembrança da sua infância. Edição Standart Brasileira das Obras Completas de Sigmund Freud. Vol. XI. Rio de Janeiro: Imago.

(1914/1996). O Moisés de Michelangelo. Edição Standart Brasileira das Obras Completas de Sigmund Freud. Vol. XIII. Rio de Janeiro: Imago.

Gil, A. C. (1991). Como elaborar projetos de pesquisas. São Paulo: Atlas.

Jung, C. G. (2001). Memórias, sonhos, reflexões. Rio de Janeiro: Nova Fronteira.

Koerich, M. S., Erdmann, A. L. (2011). O estado da arte sobre ética e saúde no Brasil: Pesquisa em banco de teses. Texto e Contexto Enfermagem, 20(3), 376-384. doi: 10.1590/S0104-07072011000300020

Laville, C., \& Dionne, J. (1999). A construção do saber: Manual de metodologia da pesquisa em Ciências Humanas. Porto Alegre: Artes Médicas.

Luna, S. V. (2002). Planejamento de Pesquisa: Uma introdução. São Paulo: EDUC.

Marconi, M. A., \& Lakatos, E. V. (1986). Técnicas de Pesquisa. São Paulo: Atlas.

Mazer, S. M., \& Melo-Silva, L. L. (2010). Identidade profissional do psicólogo: Uma revisão da produção científica no Brasil. Psicologia Ciência e Profissão, 30(2), 276-295. doi: 10.1590/S1414-98932010000200005

Moehlecke, V. (2002). O paradigma estético e a psicologia: Ressonâncias. Linhas Críticas, 8(15), 207-220. Recuperado de http://periodicos.unb.br/index. php/linhascriticas/article/view/6479/5237

Moutinho, K., \& Conti, L. D. (2010). Considerações sobre a psicologia da arte e a perspectiva narrativista. Psicologia em Estudo, 15(4), 685-694. doi: 10.1590/ S1413-73722010000400004

Nakano, T. de C., \& Wechsler, S. M. (2007). Criatividade: Características da produção científica brasileira. Avaliação Psicológica, 6(2), 261-270. 
Recuperado de http://pepsic.bvsalud.org/scielo. php?pid $=$ S1677-04712007000200015\&script $=$ sci_arttext

Nunes, M. F. O., \& Huntz, C. S. (2014). Análise da produção científica de artigos sobre lazer: Uma revisão. Psicologia Teoria e Pesquisa, 30(3), 307-315. Recuperado de http://www.scielo.br/pdf/ptp/ v30n3/08.pdf

Paglia, C. (1992). Personas Sexuais: arte e decadência de Nefertite a Emily Dickinson. São Paulo: Companhia das Letras.

Paiva, G. J. et al. (2004). Experiência religiosa e experiência estética em artistas plásticos: Perspectivas da Psicologia da Religião. Psicologia Reflexão e Crítica. 17(2), 223-232. doi: 10.1590/ S0102-79722004000200010

Pereira, M. V. (2012). O limiar da experiência estética: Contribuições para pensar um percurso de subjetivação. Pro-Posições, 1(67), 183-195. doi: 10.1590/ S0103-73072012000100012.

Perls, F. (1988). A abordagem gestáltica e testemunha ocular da terapia. Rio de Janeiro: LTC.

Raffaelli, R. (1996). Psicanálise e arte: A cruz da sublimação. Estudos de Psicologia, 13(1), 11-18. Recuperado dehttp://www.oei.es/na6034.htm

Reis, A. C. (2014). Arteterapia: A arte como instrumento no trabalho do psicólogo. Psicologia: Ciência e Profissão, 34(1), 142-157. doi: 10.1590/ S1414-98932014000100011

Rivera, T. (2006). Cinema e pulsão: Sobre "Irreversível", o trauma e a imagem. Revista do Departamento de Psicologia - UFF, 18(1), 71-76. doi: 10.1590/ S0104-80232006000100006

Silva, S. M. C. (2004). Algumas reflexões sobre a Arte e a formação do psicólogo. Psicologia Ciência e Profissão, 24(4), 100-111. doi: 10.1590/ S141498932004000400012
Silva, T. F., \& Nakano, T. de C. (2012). Criatividade no contexto educacional: Análise de publicações periódicas e trabalhos de pós-graduação na área da Psicologia. Educaşão e Pesquisa, 23(79), 257-272. doi: 10.1590/S1517-97022012005000013

Tardivo, R. C. (2012). Uma perspectiva poética-crítica em psicologia da arte. Psicologia Política, 12(23), 153-160. Recuperado de http:// pepsic.bvsalud.org/scielo.php?pid=S1519-549X2012000100011\&script=sci_arttext

Teixeira, L. C. (2005). O lugar da literatura na constituição da clínica psicanalítica em Freud. Psyche, 9(16), 115-132. Recuperado de http:// pepsic.bvsalud.org/scielo.php? pid $=$ S1415$-11382005000200008 \&$ script=sci_arttext

Vasconcellos, E. A., \& Giglio, J. S. (2007). Introdução da arte na psicoterapia: Enfoque clínico e hospitalar. Estudos de Psicologia, 24(3), 375-383. doi: 10.1590/ S0103-166X2007000300009

Vygotski, L. S. (1993). La imaginación y el arte en la infancia. Madrid: Akal.

Vygotski, L. S. (1998). Psicologia da arte. São Paulo: Martins Fontes.

Vivian, A. G., Trindade, J. (2003). Psicologia e arte: Um paradigma estético dos processos de criação. Aletheia, 17(18), 107-121. Recuperado de http:// www.redalyc.org/pdf/1150/115013455011.pdf

Zanella, A. V., \& Titon, A. P. (2005). Análise da produção científica sobre criatividade em programas brasileiros de pós-graduação em psicologia (19942001). Psicologia em Estudo, 10(2), 305-316. doi: 10.1590/S1413-73722005000200018

Recebido em: 21/08/2015

Reformulado em: 22/11/2015

Aceito em: 25/11/2015 
Sobre os autores:

André Luiz Picolli da Silva é Psicólogo e Mestre em Psicologia, Doutorando em Psicologia Clínica e Cultura pela Universidade de Brasília (UNB), Professor do Curso de Graduação em Psicologia da Universidade Federal do Sul e Sudeste do Pará (Unifesspa).

E-mail: picolli@unifesspa.edu.br

Terezinha de Camargo Viana é Professora Associada do Programa de Pós-graduação em Psicologia Clínica e Cultura, Coordenadora do Laboratório de Psicanálise e Subjetivação da Universidade de Brasília (UNB). Bolsista do CNPq.

E-mail:tcviana@unb.br

Contato com os autores:

Campus Universitário Darcy Ribeiro

Brasília-DF, Brasil

CEP: 70910-900 